\title{
A qualitative model of flashing across an aperture with a pressure differential
}

\author{
G. C. Polanco ${ }^{1}$, A. E. Holdo ${ }^{2} \&$ G. Munday ${ }^{3}$ \\ ${ }^{1}$ Universidad Simón Bolivar, \\ ${ }^{2}$ Venezuela Narvik University College, \\ ${ }^{3}$ Norway Coventry University, UK
}

\begin{abstract}
Many industries use materials that are stored above their atmospheric pressure boiling points (superheated liquids) which can form two-phase mixtures upon their accidental release to the environment at ambient conditions. The behaviour and the characteristics of these liquid-gas mixtures during a superheated liquid release due to the breaking of the metastable state can significantly affect the hazard zone and the mitigation steps that can be taken to minimize the release impact for the hydrocarbon industry or the quality of the combustion, or explosions inside the diesel engines. The dependence of the whole process over the initial parameter values as the pressure, the temperature and others factors, which can created, for some cases, a complete breaking of the liquid core into droplets at the same time that it is going out of container like unstable jet, or a complete one phase liquid jet, is recreated by an empirical model based on the flashing process similarity and a damped second order system. Results of this model agreed with some available experimental data.
\end{abstract}

Keywords: superheated liquids, empirical model, flashing process.

\section{Introduction}

Flashing is the violent phase change of a super-heated liquid when it is exposed to a pressure gradient generated by the pressure difference between the vessel or pipe line and the atmospheric pressure, due to vessels or pipelines faults. During the brief depressurisation of the liquid breaks into droplets at the same time that it is going out of container trough an opening like unstable jet, as consequence of the altering of the metastable state of the superheated liquid stored. 
Experimental research has found that for distances closer to the opening, liquid regions and large droplets are still superheated, so, they continuously break-up or evaporate in a section known as the expansion region; after this section, the droplet velocity decreases due to effects of the entrainment of air into the internal energy balance of the jet, and this new region is known as the entrainment region [8].

Flashing process is a non isentropic process in which mechanics and thermodynamics mechanisms interact generating a particular type of jet, with certain characteristics of phase velocities, temperature and mass flow. These two mechanisms work together but there is not an equal and constant predominance of any of them along the whole process. The identification of the predominance of a particular mechanism in different situations is used to characterize the type of jet formed. The fluid properties, such as, the viscosity and density, and their dependency with temperature or pressure together with the difference between the ambient conditions and the superheated initial condition of the fluid make an important contribution to the final characteristics of a flashing jet. The potential hazard of a vessels leak of metastable fluid is directly related with the fluid conditions and the mechanism predominant under certain circumstances.

\section{Qualitative model of flashing: physics considerations}

Phase change process presented in flashing needs to rich a minimum level of energy in order to appear, due to the significant difference in the internal energy of liquid and vapour phases of the same fluid. Assuming that the flashing occurs at most just outside of the opening, an analytical model of flashing across an aperture with pressure change is proposed to determine the type of jet resulting after the leak takes place.

To understand the characteristics of the different types of jet, it was necessary to establish in advance the number of cases to take into account. There are three main different types of jet, known as: liquid jet, mixture jet and gas jet with droplets $[7,9,10]$. The proposed model is based on the compilation of the individual influences of pressure differences, temperature differences and fluid properties. The pressure-temperature relationship in a fluid at saturation conditions shows that there is a variable dependence on temperature over changes in pressure for a particular pressure ratio. When the pressure increases and becomes closer to the critical point the dependence of temperature on pressure decreases. The region limited by the spinodal line, which represents the theoretical range of superheat that the fluid can achieve decreases, when the temperature or pressure values become closer to the critical condition. Larger values of temperature or pressure increase the thermal and pressure energy in the fluid, which in case of any release to ambient atmospheric conditions will need to be dissipated by the jet on its way out. The dissipation of larger quantity of energy generates a more violent phase change inside the jet because larger amount of energy have to be dissipated over a short distance. In its way, out the vessel, the fluid pressure and temperature suffer changes due to the interaction between the fluid and the ambient, from the vessel pressure value to the ambient 
pressure at sufficient long distances from the opening. When the pressure difference changes, it produces a velocity variation and liquid density variation, that can be characterized by the Reynolds number, which is the ratio of the resulting inertial force and the viscous force. The Jacob number, Ja, defined as the ratio of the energy supplied by the superheated liquid and fluid latent heat, $h_{f g}$. The ratio of the constant heat coefficient, $C_{p}$, and the latent heat drives the speed of the heat transfer phenomenon by conduction mean over evaporation phenomenon. Clearly, the Jacob number increases as a result of rising pressure due to the decrease of enthalpy and the increase of the $C_{p}$ with the pressure rise and also with the temperature difference.

The resistance of the liquid to keep itself together as a liquid jet before it stars to break-up by any external (surface instabilities or waves) or internal (nucleation) means to the jet is represented by the Weber number, defined as the ratio of inertial force and surface tension force. It can be expected that the Weber number will increase as result of the effect of the square exponent of the velocity, which increases with pressure difference, as well as, with the decrease of the surface tension with pressure rise. It suggests the existence of a critical Weber number that drives the growth of the nucleated bubbles inside the fluid [11].

The liquid density to gas density ratio can be used as measure of how much energy is necessary to overcome the phase change barrier through evaporation, which at least must correspond to latent heat of the fluid. Small density ratios indicate that less energy must be added to the system to achieve the evaporation than for higher density ratios. The boiling mechanisms inside of the fluid will depend on the introduction of sufficient kinetic energy for the molecules inside the fluid to change from liquid to vapour state.

A previous study performed by Skripov et al. [12] has confirmed that fluid properties as surface tension, specific volume, specific heat, viscosity and thermal conductivity of a superheated liquid follow the same trend as the properties at saturation point, however, the information under this condition is not often available. Due to fluid temperature will go below the boiling point only after some distance downstream of the opening within the jet, named Minimum Temperature Distance, the fluid properties at the injection temperature, $T_{i n j}$, will be delimit by the properties value at the boiling temperature, $T_{b}$, as shown by eqn (1). The values of the main properties of the liquid phase as density, $\rho_{l}$, viscosity, $\mu_{l}$, surface tension, $\sigma_{l}$, and coefficient at constant pressure, $C_{p}$, will be in all the cases limited by the value of the properties at the boiling temperature.

$$
\left.\begin{array}{l}
\rho_{l}\left(T_{i n j}\right)<\rho_{l}\left(T_{b}\right) \\
\mu_{l}\left(T_{i n j}\right)<\mu_{l}\left(T_{b}\right) \\
\sigma_{l}\left(T_{i n j}\right)<\sigma_{l}\left(T_{b}\right) \\
C_{p}\left(T_{i n j}\right)>C_{p}\left(T_{b}\right)
\end{array}\right\} \text { for } T_{i n j}>T_{b}
$$


Therefore, the properties values used to compute all non-dimensional numbers involved in a flashing problem will be assumed as the properties at the boiling point.

Resulting flashing jet also depends on geometrical considerations. The geometry of the nozzle as well as the piping system used in every experimental setting could affect the final output of the flashing jet. The diameter, the length and the shape of the nozzle have been taken as major characteristic parameters to be considered $[6,8,13,14]$. Nozzle diameter, length and shape and the wall's roughness $e$, will influence the discharge coefficient [15]. For instance, the discharge coefficient of a nozzle with sharper edges will be larger than for a nozzle with chamfered edges, and therefore, a nozzle with sharper edges will produce a larger pressure drop in the flow, as well as, larger disturbances in the flow field.

The nozzle diameter also affects total mass flow rate discharged and the area available to conduct the heat transfer inside the fluid. The cross sectional area of the fluid is drastically reduced from inside the tank to the exit passing through the nozzle. The smaller nozzle diameter will cause a reduction of the fluid heat transfer area, making it difficult for the fluid to keep the same conduction heat transfer rate on its way out, augmenting the possibilities of a violent and explosive flashing process. The nozzle length determines the period of time required by a fluid with certain velocity to flow trough the nozzle. It can promote the formation of heterogeneous nuclei inside the nozzle due to the increase of the contact between the fluid and the internal surface irregularities of the nozzle [16]. If, it is the case that the fluid can still be liquid at the exit of the nozzle, then the length will affect the type and size of perturbation or instabilities presented in the jet surface, as product of the internal flow pattern. In cases of two-phase discharges the length is an important factor in the determination of the two phase flow pattern inside a pipe [6, 17-19].

\section{Model's development}

It is seen that nucleation has a significant effect on flashing as the initial process that produces the breaking of the liquid continuum [20]. Nuclei are the starting point of gas bubbles inside the liquid and they are in turn responsible for the liquid transformation into droplets after the release. The total nucleation is responsible for all the vapour in the jet [21, 22].

The proposed model is a one dimensional model based on a second order system which accounts for damping forces and stiffness forces over the nucleation process of a system driven by temperature and pressure differences at conditions where phase change occurs for liquids [23].

There are similarities between the parameters that influence the nuclei generation process and the physical parameters that take part in the motion generation within a damped second order system and the type of response that the system can have under certain circumstances. For instance, both systems need to achieve a minimum level of energy to initiate the process, the type of response of the whole system to the velocity of the changes, the behaviour of the 
system will change drastically depending on the output of the system. It possible to think that, every case of leakage has the possibility to experience nucleation at different levels: explosive, very weak or none. The possibilities of the actual system response will be determined by the interaction between the parameters involved in the stiffness coefficient and the dissipation coefficient.

Based on those similarities the variable to be considered as the modelled parameter for the model proposed is the number of bubble clusters generated, called $N$. The nucleation rate of bubble clusters $\dot{N}$, is determined from the balance of those parameters by the second derivative of the numbers of nuclei, $\ddot{N}$. The letters $K^{*}$ and $C^{*}$ of eqn (2) represent the stiffness and damping coefficient by unit mass. The stiffness coefficient per unit mass and damping coefficient per unit mass will be named here after as the production coefficient and the dissipation coefficient of the nucleation system. So, the left hand side term of the eqn (1) represents the number of bubbles per squared second instead that the acceleration defined as distance per squared second.

$$
\ddot{N}=-C^{*} \dot{N}-K^{*} N
$$

The model development was centred in the determination of the equivalent coefficients to the spring and the damped component of a damped second order system. The production coefficient represents the interaction of all parameters that potentially promote of the nucleation process. The potential of the liquid to nucleate is assumed to be a function of the degree of superheat, pressure difference, diameter and length of the nozzle as well as properties of the fluid. However, the relative magnitudes of the temperature and the pressure of the fluid with respect to the critical parameters of the flow are also important variables to take into account. The critical point represents the maximum potential energy contained in the flow. The closer the fluid is to the critical conditions, the lesser energy is needed to produce a phase change. Whilst, the dissipation coefficient represents the influence of the parameters that tries to slow down the nucleation. The influence of a particular variable is not exclusively in favour or against nucleation, there are some variables having a relevant role in both aspects. As result of the analysis of experiment data it is clear that the influence of a particular variable depends on its proportion respect to a reference value, as for instance the critical conditions values of a substance or the standard atmospheric conditions. The expression developed for these two coefficients are represented in eqns (3) and (4).

$$
\begin{aligned}
& K^{*}=g_{o} C_{D} d^{1.25} \frac{l}{d}\left(p_{i n j}-p_{o}\right) \sqrt{\frac{T_{i n j}-T_{b}}{T_{i n j}}} \frac{1}{\rho_{g}} \\
& C^{*}=g_{1} T_{b} \frac{d}{l}+ \\
& g_{2} \frac{\rho_{l i q}}{\rho_{g a s}} \frac{p_{c}}{p_{o}} \frac{e}{d} \frac{\left(T_{c}-T_{i n j}\right)}{T_{i n j}} \frac{C p\left(T_{c}-T_{i n j}\right)}{L}\left(\frac{\sigma}{\mu} \sqrt{\frac{\rho_{l}}{2 p_{0}}}\right) \frac{\left(p_{c}-p_{i n j}\right)}{\rho_{g}} \\
& \frac{\left(p_{c}-p_{i n j}\right)}{L}
\end{aligned}
$$


Note that production coefficient as natural number is only defined for temperatures higher that the boiling temperature.

The criterion used to establish the characteristics of the resulting type of jet is the same used in a damped second order system which is given by the resolution of the eqn (2). Then, when a leak takes place the system is suddenly exposed to a pressure gradient between the inside and outside of the vessel. The answer of the system can be identified as under damped, damped or over damped. Every condition is associated with an equation that shows the relationship between the production coefficient $K^{*}$ and dissipation coefficient $C^{*}$, as shown in eqns (5), (6) and (7), respectively.

An over-damped system the liquid will stay liquid even after the nozzle. It is represented by eqn (5):

$$
4 K^{*}<C^{* 2} \text { over damped }
$$

A damped system will correspond to the case where the liquid has vapour bubbles inside of the core region is present after the nozzle. It is represented by eqn (6):

$$
4 K^{*}=C^{* 2} \text { damped }
$$

An under damped system will correspond to the full atomized case, where the remaining liquid is present as droplets after the nozzle. It is represented by eqn (7):

$$
4 \mathrm{~K}^{*}>\mathrm{C}^{* 2} \quad \text { under damped }
$$

The definition of the sign parameter is introduced to normalize the output of the model. A positive unity value of sign parameter means the system achieves flashing condition and a negative unity value means the system do not achieve flashing condition.

$$
\operatorname{sign}=\frac{4 K^{*}-C^{* 2}}{a b s\left(4 K^{*}-C^{* 2}\right)}
$$

To finish the model, the incorporation of three dimensional constant named $g_{0}, g_{1}$ and $g_{2}$ expressed in eqns (3) and (4) was needed. The units of the constants are consistent with the complete equation of balance of nucleation, nucleation second and first derivative and the nucleation itself. The values of these constants are the product of an empirical and numerical work of the experimental set of data available for hydrocarbons and water [6, 8, 11, 13, 24-28].

The nucleation is expressed by the number of nuclei created and considering that it is not a proper unit then the units of the whole equation will be number of nuclei per squared second. Then, the unit of the production coefficient is the inverse squared second and the unit of the dissipation coefficient is the inverse of second. The numerical values were deducted taken all parameters involved in the international system unit (SI). The values are quite close each other due to the intension of the model is basically to catch the behaviour of the different fluid on the actual expression of every term rather that the numerical values of the constants used [23]. 


\section{Results}

In this section the results corresponding to two different cases of hydrocarbons are shown. The first case cover the experiment made by Johnson and Woodward [9] using chlorine as working fluid.

The first three columns of the Table 1 contain the original for the first case and the fourth column contains the results of the application of the sign parameter, eqn (8), for all the chlorine cases tested. This set of data allows testing the influence of the pressure, the pressure, and the effects of the nozzle characteristics on the release exit. The sign parameter corresponding to the cases of temperature of $289.63 \mathrm{~K}$ did not appear, due to the production and dissipation coefficients were not defined for temperature values smaller than the boiling temperature of the fluid.

Table 1: $\quad$ Model results for CFC11. Source: [9].

\begin{tabular}{|c|c|c|c|}
\hline $\begin{array}{l}\text { Pressure } \\
{[\mathrm{kPa}]}\end{array}$ & $\begin{array}{c}\text { Temperat } \\
\text { ure }[\mathrm{K}]\end{array}$ & Observations & sign \\
\hline 161,800 & 297.90 & $\begin{array}{l}\text { Liquid stream remained together until vertical } \\
\text { movement of stream was dominant. }\end{array}$ & 1.0 \\
\hline 163,500 & 308.95 & No break-up of liquid stream visible & 1.0 \\
\hline 168,100 & 289.63 & $\begin{array}{l}\text { Liquid stream breaks up during vertical portion of } \\
\text { trajectory into large drops and globs of liquid. } \\
\text { Considerable splashing of liquid when it contacts } \\
\text { the capture surface. }\end{array}$ & - \\
\hline 190,400 & 314.40 & $\begin{array}{c}\text { Liquid stream begins to break up about } 0.3 \mathrm{~m} \text { from } \\
\text { release point }\end{array}$ & 1.0 \\
\hline 224,100 & 319.94 & $\begin{array}{l}\text { Near the release point, the stream appears thicker } \\
\text { and slightly more broken up. }\end{array}$ & 1.0 \\
\hline 254,900 & 324.77 & Stream appears more broken up from $2 \mathrm{~m}$ on. & 1.0 \\
\hline 269,700 & 327.32 & $\begin{array}{l}\text { No vapour pockets were visible in stream. Break-up } \\
\text { of liquid stream occurred, but more uniformly than } \\
330 \mathrm{~K} \text { liquid. }\end{array}$ & 1.0 \\
\hline 302,000 & 330.71 & $\begin{array}{l}\text { Definite liquid break-up about } 0.2 \mathrm{~m} \text { from release } \\
\text { point. }\end{array}$ & -1.0 \\
\hline 362,500 & 338.37 & $\begin{array}{l}\text { Majority of liquid stream is being blown apart by } \\
\text { vapour formation. The entire stream is broken apart } \\
\text { about } 20 \text { percent of the time. }\end{array}$ & -1.0 \\
\hline 366,700 & 338.42 & $\begin{array}{l}\text { Liquid stream begins to break-up near the release } \\
\text { point. Complete shattering of the stream occurs } \\
\text { occasionally. }\end{array}$ & -1.0 \\
\hline 392,700 & 341.09 & $\begin{array}{l}\text { Stream break-up occurs about } 2-3 \mathrm{~cm} \text { from release } \\
\text { point. }\end{array}$ & -1.0 \\
\hline 470,600 & 348.81 & $\begin{array}{l}\text { Liquid stream has completely broken up. Visible } \\
\text { drops appear infrequently. }\end{array}$ & -1.0 \\
\hline 554,100 & 354.96 & $\begin{array}{l}\text { No large drops visible. Complete stream break-up } \\
\text { within } 1-2 \mathrm{~cm}(0.5 \text {-inch }) \text { of release point. }\end{array}$ & -1.0 \\
\hline
\end{tabular}


Figure 1 shows the influence of the temperature of the type if jet generated after the nozzle, since a complete liquid jet to a fully sprayed one. Figure 2 shows the pressure effect on the jet type. Although both jets contain droplets after the nozzle, the spray angle is larger for the larger pressure. Figure 3 shows the effects on the jet of the change in diameter of the nozzle. In this particular case it can be see that the jet flashing location becomes closer to the nozzle, changing the profile at the exit of the nozzle since a liquid jet discharge to a complete sprayed jet.

The effect of the length of the nozzle, is normally represented by the length diameter ratio, instead the actual value. Figure 4 shows three cases of $850 \mathrm{kPa}$ and $20^{\circ} \mathrm{C}$ for $\mathrm{L} / \mathrm{D}, 0,2$ and 7 respectively.

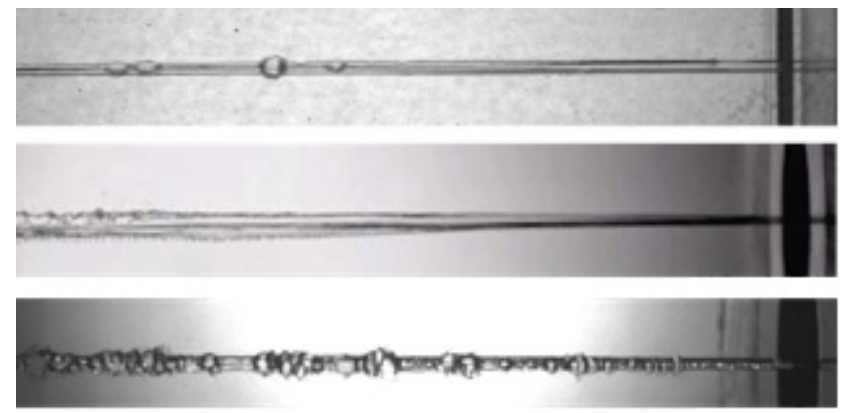

$$
\begin{aligned}
& \text { sign }=-1 \\
& \text { sign }=-1 \\
& \text { sign }=-1 \\
& \text { sign }=-1
\end{aligned}
$$

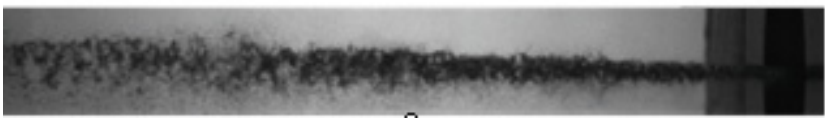

Figure 1: Photograph of a flashing jet from a nozzle diameter of $1 \mathrm{~mm}$ and fixed pressure of $85.000 \mathrm{KPa}$, showing the test corresponding to five distinct temperatures, $13,14,18.5$ and $20.2^{\circ} \mathrm{C}$ respectively. Source: [5].

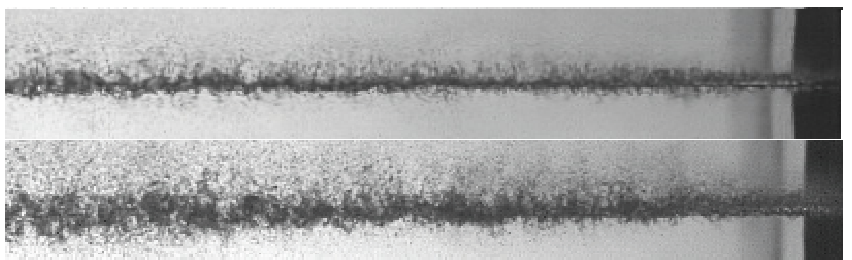

sign $=-1$

$\operatorname{sign}=-1$

Figure 2: $\quad$ The effects of two pressure values, $850 \mathrm{kPa}$ and $1250 \mathrm{~Pa}$ in a flashing jet. Temperature $=20^{\circ} \mathrm{C}$, nozzle diameter $=1 \mathrm{~mm}$. Source: $[5]$. 


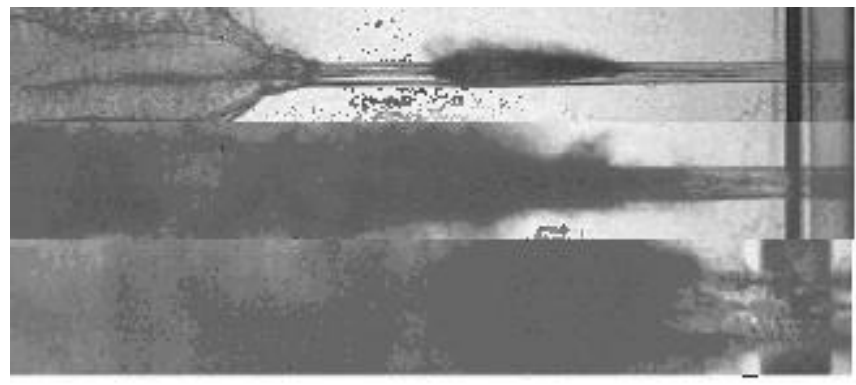

$$
\begin{aligned}
& \operatorname{sig} n=-1 \\
& \operatorname{sig} n=-1 \\
& \operatorname{sig} n=-1
\end{aligned}
$$

Figure 3: $\quad$ Flashing jet from three nozzle diameters $(2 \mathrm{~mm}, 3 \mathrm{~mm}$ and $4 \mathrm{~mm}$ respectively). All cases tested at $850 \mathrm{kPa}$ and $13^{\circ} \mathrm{C}$. Source: [5].

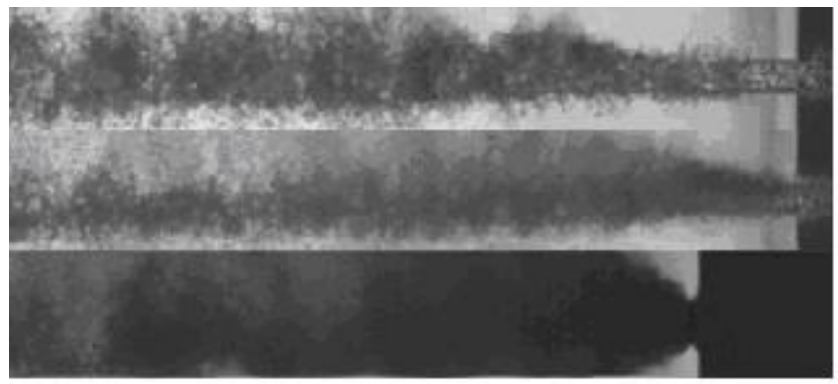

$$
\begin{aligned}
& \operatorname{sig} n=-1 \\
& \operatorname{sig} n=-1 \\
& \operatorname{sig} n=-1
\end{aligned}
$$

Figure 4: Flashing jet from a nozzle of $2 \mathrm{~mm}, 850 \mathrm{kPa}$ and $20^{\circ} \mathrm{C}$ for $\mathrm{L} / \mathrm{D}=0$, 2 and 7 respectively. Source: [5].

The type of regime achieve by the fluid after the release is an indication of how strong the nucleation rate was inside the fluid. The stronger the nucleation is, the breaking of the liquid core will be more extreme and the droplets generated tend to be smaller.

\section{Discussions}

The general trend of the experimental information is reproduced by the present model. The information shown in Table 1 highlights the quality of the obtained results. The output of the model reproduces the experimental observations reported in the original work.

The present model predicts that all the cases of R134a will produce a flashing jet. However, the experimental evidence is, in some cases, ambiguous. The criterion will depend on the accuracy in identifying a liquid with visible bubbles inside the jet or a complete liquid jet. It is important to mention that the order of magnitude of the reduced pressure range evaluated for the R134a cases is about 0.20 after . This value of reduced pressure is larger than any other experimental setting, where the common range for the evaluated reduced pressure is about 0.04. Therefore the dissipation coefficient has a smaller value which is overcome 
for the production coefficient. The model is capable of reproducing the experimental observations related to basic influences of diameter increases, pressure increase, length increase, and temperature increase.

A good agreement was also obtained for experiments using water, . The model fails to predict the liquid discharges corresponding to the data reported by Miyatake et al. [14]. The discrepancies between the result and the observations reported can be related to presence of a large length diameter ratio, up to a maximum of $L / D=78.2$. When, the length of the nozzle becomes large in comparison with the diameter, then the temperature effect becomes stronger since the pressure energy is mainly used to overcome the viscous losses through the nozzle and the viscous forces in the nozzle walls affects the force balance in the fluid. This alters the physics of the system modelled. In this case the surface tension will not be the major force acting on nucleation. Therefore, the assumptions used to develop the expression of the production and dissipation coefficients do not apply in a correct way for this experimental data.

\section{Conclusions}

A one dimensional model of flashing from an aperture has been developed. This model uses the nucleation process as the major process within flashing jet. This model called production dissipation model covers the study of flashing occurs it or not, and it is capable of reproducing the effects on the jet of the change of diameter and length of the nozzle, as well as, the variation in temperature and pressure and their relative position to the critical temperature or pressure of the fluid.

The occurrence of a flashing jet can be determined by the model based on the force balance between the promoting forces and dissipation forces of nucleation, in a similar way to a second order damped system. This balance take into account individual effect of the problem parameters, such as initial pressure and temperature as well as geometry dimensions represented by dimensionless parameters as Reynolds number, Jacob number, Weber number, ratio of density, friction factors or discharge coefficient. This model can be used for both water and hydrocarbons fluids. The solution of the model will determine the behaviour of the system as sub-damped (flashing jet), damped or over damped system (liquid jet).

The model allows the inclusion of the friction factor or dimensionless roughness, as important parameter in the nucleation process sue to its important as a potential nuclei sources, which are not normally included in any other work presented in the literature.

If was found that the different behaviour of water and hydrocarbon is based on the appreciable difference of their properties. In particular the liquid density and gas density ratio of hydrocarbons and water, which represents the quantity of energy needed to overcome the phase change barrier by evaporation and differences in surface tension. 


\section{References}

[1] Deaves, D.M., et al., Modelling of catastrophic flashing releases. Journal of Hazardous Materials (A88): p. 1-32, 2001.

[2] Michaella, M., N. Piccinini, and A. Poggio, Analysis of an LPG accidental release. Process Safety and Environmental Protection. 82: p. 128-131, 2004.

[3] Lee, C.S. and S.W. Park, An experimental and numerical study on fuel atomization characteristics on high-pressure diesel injection sprays. Fuel. 81: p. 2417-2423, 2002.

[4] Diek, H. and R.L. Roberts, The determination of the sauter mean droplet diameter in fuel nozzle sprays. Applied optics. 9(9): p. 2007-2014, 1970.

[5] Yildiz, D., P. Rambaud, and J.v. Beek. Break-up, droplet size and velocity characterisation of a two-phase flashing R134A jet. in 5th International Conference on Multiphase Flow. Yokohama, Japan. 2004.

[6] Park, B.S. and S.Y. Lee, An experimental investigation of the flash atomization mechanism. Atomatization and Sprays. 4: p. 159-179, 1994.

[7] Peter, E.M., A. Takimoto, and Y. Hayashi, Flashing and shattering phenomena of superheated liquid jets. JSME International Journal. 37(2): p. 313-321, 1994.

[8] Yildiz, D., Dynamics of two-phase flashing jets. VKI Lectures Series. Belgium: von Karman Institute for Fluid Dynamic. 2003

[9] Johnson, D.W. and J.L. Woodward, RELEASE A Model with data to predict aerosol rainout in accidental releases. Center for chemical process safety. New York: American Institute of Chemical Engineers. 1999

[10] Lin, S.P. and R.D. Reitz, Drop and spray formation from liquid jet. Annual Review Fluid Mechanics. 30: p. 85-105, 1998.

[11] Brown, R. and L. York, Sprays formed by flashing jets. American Institute of Chemical Engineers. 8(2): p. 149-153, 1962.

[12] Skripov, V.P., et al., Thermophysical properties of liquids in metaestable (superheated) state. Amsterdam: Gordon and Breach Science Publisher. 1988

[13] Reitz, R.D., A photographic study of flashing-boiling atomization. Aerosol, Science and Technology. 12: p. 561-569, 1990.

[14] Miyatake, O., et al., An experimental study of spray flash evaporation. Desalination. 36(2): p. 113-128, 1981.

[15] Potter, M., D. Wiggert, and M. Hondzo, Mechanics of fluids. Second Edition ed: Prentice Hall Inc. 1997

[16] Yan, F. and M. Giot, A nucleation model for superheated liquids in adiabatic vessel and pipes. p. 323-331, 1989.

[17] Barnea, D., O. Shoham, and Y. Taitel, Flow pattern transition for downward inclined two phase flow; horizontal to vertical. Chemical Engineering Science. 37(5): p. 735-740, 1982.

[18] Barnea, D., O. Shoham, and Y. Taitel, Flow pattern transition for vertical downward two phase flow. Chemical Engineering Science. 37(5): p. 741744, 1982. 
[19] Barnea, D., et al., Flow pattern transition for gas-liquid flow in horizontal and inclined pipes. Comparison of experimental data with theory. International Journal of Multiphase Flow. 6(3): p. 217-225, 1980.

[20] C Cartes, M.G.C.a.R.S., Van der waals normal form for a one-dimensional hydrodynamic model. Physics Review. 70(031302-1/8), 2004.

[21] R Ramanathan, Y.K.M., K. K. Gupta, Agam K Jha and S. S. Singh, A comparative study of two models of QCP-Fireball formation. eprint arXiv:hep-ph/0502046, 2005.

[22] D. L. Frost, R.B., and J. Nerenberg, TP 12123E. Small-Scale BLEVE Tests with Refrigerant-22. 1995, Transportation Development Centre: Quebec. p. 48.

[23] Polanco, G., Phase change within flows from breaches of liquefied gas pipelines. 2008, Coventry: Coventry.

[24] Solomon, et al., Flow and atomization in flashing injectors. Atomization Spray Technology. 1: p. 53-76, 1985.

[25] Hervieu, H. and T. Veneau, Experimental determination of the droplet size and velocity distributions at the exit of the bottom discharge pipe of a liquefied propane storage tank during a sudden. Journal of Loss Prevention in the Process Industries. 9(6): p. 413-425, 1996.

[26] Bolle, L.a.D.-Z., P., Experimental and theoretical analysis of flashing water flow through a safety valve. Journal of Hazardous Materials. Vol. 46.: p. pp. 105-116, 1996.

[27] Gemci, T., et al., Experimental study of flash atomization of binary hydrocarbon liquid. International Journal of Multiphase Flow. 30: p. 395417, 2004.

[28] Gemci, T., et al., Flash atomization of water / acetone solutions. Atomization and Sprays. 14: p. 459-475, 2004. 